\title{
Prevalence and progression of aortic root dilatation in highly trained young athletes
}

\author{
Sabiha Gati, ${ }^{1}$ Aneil Malhotra, ${ }_{1}^{1}$ Catherine Sedgwick, ${ }^{1}$ Nikolaos Papamichael, ${ }^{2}$ \\ Harshil Dhutia, ${ }^{1}$ Rajan Sharma, ${ }^{1}$ Anne H Child, ${ }^{1}$ Michael Papadakis, ${ }^{1}$ Sanjay Sharma ${ }^{1}$
}

${ }^{1}$ Cardiology Clinical and Academic Group, St. George's, University of London, London. SW17 ORE, UK

${ }^{2}$ Queen Elizabeth Hospital, London, UK

\section{Correspondence to} Professor Sanjay Sharma, Cardiology clinical academic group, St. George's, University of London, Cranmer Terrace, London SW17 ORE, UK; ssharma21@hotmail.com

MP and SS contributed equally.

Received 22 October 2018 Revised 10 January 2019 Accepted 15 January 2019 Published Online First 25 March 2019

\section{Linked}

http://dx.doi.org/10.1136/ heartjnl-2019-314759

\section{Check for updates}

(C) Author(s) (or their employer(s)) 2019. No commercial re-use. See rights and permissions. Published by BMJ.

To cite: Gati S, Malhotra A, Sedgwick $C$, et al. Heart 2019:105:920-925.

\section{ABSTRACT}

Objectives Aortic root dilatation is reported in young athletes; however, it is unclear whether such remodelling is physiological or, whether it represents a potential aortopathy. This observational study investigated the prevalence and progression of aortic root dilatation in young athletes competing at regional or national level. Methods Between 2003 and 2015, 3781 athletes aged $19 \pm 5.9$ years $(63.3 \%$ male) underwent echocardiography as part of a cardiac screening programme to identify athletes with structural abnormalities. Athletes trained for an average of 16.7 hours per week. Aortic diameter was measured at the level of sinuses of Valsalva. Results were compared with 806 controls. Athletes with an enlarged aortic diameter were followed up for $5 \pm 1.5$ years.

Results Athletes revealed a larger mean aortic diameter compared with controls $(28.3 \pm 4.1$ vs $27.8 \pm 4.1 \mathrm{~mm}$; $p=0.01$ ). The 99th percentile value for aortic diameter in the athlete cohort was defined as the upper limit and was $40 \mathrm{~mm}$ in males and $38 \mathrm{~mm}$ in females. The aortic diameter measured $>40 \mathrm{~mm}$ in five male $(0.17 \%)$ $(40-43 \mathrm{~mm})$ and $>38 \mathrm{~mm}$ in six female $(0.4 \%)$ (39$41 \mathrm{~mm}$ ) athletes. During follow-up, none of the athletes with an enlarged aortic diameter showed progressive aortic enlargement compared with the first assessment $(40.6 \pm 0.9$ vs $40.5 \pm 0.7 \mathrm{~mm}$ in males; $(p=0.111)$ and $38.3 \pm 0.6$ vs $38.0 \pm 0.7 \mathrm{~mm}$ in females; $(p=0.275))$.

Conclusions A small minority $(0.3 \%)$ of athletes reveal an enlarged aortic diameter. Medium-term follow-up does not reveal progressive enlargement of the aortic diameter indicative of aortopathy. Longer surveillance studies are necessary to elucidate the precise significance of an enlarged aortic diameter in athletes.

\section{INTRODUCTION}

Studies in highly trained athletes have revealed increased aortic dimensions compared with sedentary controls. ${ }^{1-4}$ Existing data from a large cohort of Italian athletes showed that an aortic root exceeding $\geq 40 \mathrm{~mm}$ in men and $\geq 34 \mathrm{~mm}$ in women is rare ${ }^{1}$ and forms the basis of the current definition of physiological upper limits in athletes. ${ }^{5}$ However, it is still unclear whether aortic root dilatation exceeding these limits represents a physiological response to the haemodynamic stress of exercise or a potential aortopathy. The American Heart Association/American College of Cardiology (AHA/ ACC) advises that athletes with mild aortic root enlargement $(40-41 \mathrm{~mm}$ in males and $36-38 \mathrm{~mm}$ in females) should be assessed on a 6-12 monthly basis for progressive root enlargement. ${ }^{6}$ Although these recommendations are a useful guide for physicians, they are generally informed by a very small number of studies ${ }^{146}$ and only one study has performed adequate follow-up of athletes with an enlarged aortic diameter. ${ }^{1}$ We investigated the prevalence and progression of an enlarged aortic root dilatation in highly trained athletes.

\section{METHODS \\ Athletes}

Between 2003 and 2015, 3850 young athletes aged 14-35 years underwent cardiac evaluation as part of a preparticipation cardiac screening programme. All athletes competed at regional or national level. Cardiac evaluation consisted of a self-reported health questionnaire pertaining to symptoms, a personal history of previous hypertension and pregnancy, and a family history of Marfan syndrome, premature cardiovascular disease or sudden cardiac death, cardiovascular examination, 12-lead ECG and two-dimensional echocardiography. Sixty-nine athletes $(1.8 \%)$ were excluded due to a blood pressure $(\mathrm{BP})>140 / 90 \mathrm{~mm} \mathrm{Hg}$ on three consecutive occasions $(n=14)$, bicuspid aortic valve $(n=46)$, established atrial septal defects $(n=6)$, features consistent with hypertrophic cardiomyopathy $(n=2)$. One cricket player had an aortic root diameter of $52 \mathrm{~mm}$ and underwent urgent surgery. The final population consisted of 3781 athletes (2393 males and 1388 females). Athletes engaged in 38 different sporting disciplines, which were categorised as 'predominantly static', 'predominantly endurance' and 'mixed sports'. The results were compared with healthy controls.

\section{Controls}

The control population consisted of 806 young sedentary volunteers aged 14-35 years who were recruited from a population screening programme offered by the charitable organisation Cardiac Risk in the Young. ${ }^{7}$ Inclusion criteria consisted of a sedentary lifestyle, defined as physical activity $\leq 3$ hours per week; absence of cardiac symptoms or a family history of premature cardiovascular disease, thoracic aortic aneurysms or sudden cardiac death; BP <140/90 mm Hg and a structurally normal heart.

\section{Echocardiography}

Two-dimensional echocardiography was performed using either the Philips Sonos 7500, Philips iE33 
or Philips CPX50 (Bothell, Washington, USA). Standard views were obtained and analysed according to protocols specified by American Society of Echocardiography. ${ }^{8}$ Transverse aortic root dimensions were measured from the parasternal long axis view in end-diastole, at the level of the sinuses of Valsalva. Measurements were made from leading edge to leading edge, and averaged over three consecutive cycles. ${ }^{9}$ The aortic valve was evaluated on the two-dimensional images, including parasternal long and short axis views. Three hundred twenty random cardiac measurements were repeated on a separate occasion by the first author (SG) and independently by another author (NP) to assess intraobserver and interobserver variability, respectively.

\section{Definition of aortic enlargement}

Similarly to a previous large study, ${ }^{1}$ we chose the 99 th percentile from the mean of the absolute aortic diameter in our study population to define an abnormally enlarged aortic root dimension. We also calculated the $\mathrm{Z}$ score using the Devereux equation. ${ }^{10} \mathrm{~A}$ $\mathrm{Z}$ score $>3$ was considered abnormal.

\section{Further evaluation and follow-up}

Athletes with an abnormally enlarged aortic root were evaluated for skeletal and ocular abnormalities in accordance with Ghent criteria for Marfan syndrome and were followed up annually. ${ }^{11}$ The mean follow-up was $5 \pm 1.5$ years (range $3-7$ years).

\section{Data analysis and statistics}

Statistical analyses were performed using SPSS V.18.0 (SPSS, Chicago, Illinois, USA). Values are expressed as mean \pm SD or percentages, as appropriate. Variables were tested for normality using the Kolmogorov-Smirnov test. Differences between group means were compared using independent t-test or MannWhitney $U$ test (for normally and non-normally distributed variables, respectively). Chi-squared test was used to test group differences of proportions. Multiple-adjusted linear regression was performed to identify independent predictors of aortic root diameter in athletes including age, body surface area (BSA), ethnicity, gender height, heart rate, systolic BP, diastolic $\mathrm{BP}$, left atrial diameter, left ventricular end-diastolic diameter (LVEDD) left ventricular (LV) mass, maximal-LV wall thickness and years of training. One-way analysis of variance was used to compare the aortic root dimensions between groups of athletes with different types of exercise. Repeated measures analysis of variance was performed to evaluate changes of the aortic root dimensions during the follow-up period. Statistical significance was defined as a two-tailed $\mathrm{p}$ value of $<0.05$ throughout.

\section{Study ethics and consent}

Written consent was obtained from individuals aged 16 years or over and from a parent/guardian for those aged $<16$ years.

\section{RESULTS}

Demographics

Athletes

Athletes were aged $19.9 \pm 5.9$ years. The majority were male (table 1). Athletes trained for an average of 16.7 hours per week. The majority (58\%) engaged in sporting disciplines combining a mixture of static and endurance components, $25 \%$ participated in predominantly endurance sports and $17 \%$ in predominantly static sports; $52 \%$ of athletes participated in either soccer, tennis, swimming or rugby (online supplementary table 1).
Table 1 Comparison of demographics between athletes and controls*

\begin{tabular}{lllc}
\hline Parameters & Athletes $(\mathrm{n}=3781)$ & Controls $(\mathrm{n}=806)$ & P value \\
\hline Age (years) & $19.9 \pm 5.9$ & $20.3 \pm 6.2$ & 0.109 \\
Body surface area $\left(\mathrm{m}^{2}\right)$ & $1.8 \pm 0.3$ & $1.8 \pm 0.2$ & 0.062 \\
Height $(\mathrm{cm})$ & $174.8 \pm 11.5$ & $172.6 \pm 9.8$ & $<0.001$ \\
$\begin{array}{l}\text { Systolic blood pressure }(\mathrm{mm} \\
\mathrm{Hg})\end{array}$ & $112 \pm 12.9$ & $124 \pm 13.4$ & $<0.001$ \\
Sex $\mathrm{n}(\%)$ & & & \\
$\quad$ Males & 63.3 & 61.0 & 0.228 \\
$\quad$ Females & 36.7 & 39.0 & \\
Ethnicity $(\%)$ & & & \\
$\quad$ Caucasian & 88.8 & 75.7 & \\
$\quad$ African/Afro-Caribbean & 6.2 & 19.9 & \\
Asian & 1.7 & 3.5 & \\
Mixed race & 2.7 & 0.7 & \\
$\quad$ Other ethnicity & 0.7 & 0.2 & \\
Hours of training/week & $16.7 \pm 8.1$ & $2.7 \pm 3.1$ & \\
\hline *Data expressed as mean \pm SD. & &
\end{tabular}

\section{Controls}

The majority of controls were white males, and were of a similar age to the athletes. Body mass index (BMI) values were not statistically different between athletes and controls. However, a significant difference in height was noted $(174.83 \pm 11.52$ vs $172.66 \pm 9.83 \mathrm{~cm}, \mathrm{p}<0.001)$. None of the controls exhibited features suggestive of Marfan syndrome.

\section{Aortic root diameter in athletes}

Absolute aortic dimensions

Athletes revealed a lower resting heart rate and large LV dimensions and LV mass compared with control (table 2). Athletes exhibited a larger mean aortic root diameter compared with controls $(28.3 \pm 4.1 \mathrm{~mm}$; range $17-43$ vs $27.8 \pm 4.1 \mathrm{~mm}$; range $17-40 \mathrm{~mm} ; \mathrm{p}=0.010$ ) (figure 1). The 99th percentile value for aortic root diameter in control males and females was 38 and $36 \mathrm{~mm}$, respectively. Based on these values, 58 (2.4\%) male athletes and $32(2.3 \%)$ female athletes would have been diagnosed with an enlarged aortic diameter.

Male athletes revealed a larger aortic root diameter compared with female athletes $(29.3 \pm 4.0 \mathrm{~mm}$; range $17-43$ vs $26.6 \pm 3.9 \mathrm{~mm}$; range $17-40 \mathrm{~mm}, \mathrm{p}<0.001)$. The 99 th percentile for athletic males and females was 40 and $38 \mathrm{~mm}$, respectively. Athletes engaging in predominantly endurance sports revealed a trend towards a larger aortic diameter compared with athletes performing mixed sports and purely static sports (table 3), but this did not achieve statistical significance. The aortic diameter did not exceed $>43 \mathrm{~mm}$ in male athletes or $>40 \mathrm{~mm}$ in female athletes.

\section{Indexed aortic dimensions}

There were no differences between athletes and controls when the aortic diameter was indexed for BSA $\left(15.5 \pm 2.0 \mathrm{~mm} / \mathrm{m}^{2}\right.$ (range $8.5-26.0 \mathrm{~mm} / \mathrm{m}^{2}$ ) vs $15.4 \pm 2.0 \mathrm{~mm} / \mathrm{m}^{2}$ (range $13.8-$ $\left.25.1 \mathrm{~mm} / \mathrm{m}^{2}\right) ; \mathrm{p}=0.215$ ). Similarly, there were no differences between athletic males and females when aortic dimensions were indexed for BSA $\left(15.5 \pm 2.0 \mathrm{~mm} / \mathrm{m}^{2}\right.$ (range $8.5-26.0 \mathrm{~mm} / \mathrm{m}^{2}$ ) vs $15.4 \pm 2.1 \mathrm{~mm} / \mathrm{m}^{2}$ (range $\left.9.7-24.0 \mathrm{~mm} / \mathrm{m}^{2}\right) ; \mathrm{p}=0.146$ ). The 99th percentile value for the indexed aortic dimensions in athletic men and women was 22.3 and $22.1 \mathrm{~mm} / \mathrm{m}^{2}$, respectively. 
Table 2 Echocardiographic comparison between athletes and controls* This table is redundant and needs to come out. We do not make reference to it and the most important information (lines 3 and 4) is already in the text.

\begin{tabular}{lllr}
\hline & Athletes $(\mathrm{n}=3781)$ & Controls $(\mathrm{n}=806)$ & P value \\
\hline HR $(\mathrm{bpm})$ & $60.6 \pm 11.9$ & $71.7 \pm 14.4$ & $<0.001$ \\
Ao $(\mathrm{mm})$ & $28.3 \pm 4.1$ & $27.8 \pm 4.1$ & 0.010 \\
Ao $\left(\mathrm{mm} / \mathrm{m}^{2}\right)$ & $15.5 \pm 2.1$ & $15.4 \pm 2.0$ & 0.019 \\
Max-LVWT $(\mathrm{mm})$ & $9.2 \pm 1.3$ & $8.8 \pm 1.2$ & $<0.001$ \\
LVED $(\mathrm{mm})$ & $51.0 \pm 5.2$ & $48.2 \pm 4.8$ & $<0.001$ \\
LVM/BSA $\left(\mathrm{g} / \mathrm{m}^{2}\right)$ & $113 \pm 32$ & $106 \pm 38.1$ & $<0.001$ \\
LAD $(\mathrm{mm})$ & $34.0 \pm 6.0$ & $31.7 \pm 8.7$ & $<0.001$ \\
FS $\%$ & $34.9 \pm 5.7$ & $36.0 \pm 5.9$ & 0.004 \\
AV Vmax $(\mathrm{cm} / \mathrm{s})$ & $1.36 \pm 2.4$ & $1.49 \pm 4.1$ & 0.101 \\
E/A ratio & $2.2 \pm 0.8$ & $2.1 \pm 0.6$ & 0.223 \\
\hline
\end{tabular}

${ }^{*}$ Data are expressed as mean \pm SD.

Ao, aortic sinus of Valsalva diameter; AV Vmax, continuous wave aortic flow velocity; E/A, ratio of early diastolic mitral valve peak inflow velocity to late diastolic mitral valve inflow velocity; HR, heart rate; LA, left atrial diameter; LV FS, left ventricular fractional shortening; LVED, LV end-diastolic diameter; Max-LVWT, maximal left ventricular wall thickness in end diastole.

Athletes with absolute aortic enlargement

The aortic root measured $>40 \mathrm{~mm}$ (range $41-43 \mathrm{~mm}$ ) in 5 $(0.17 \%)$ male athletes and $>38 \mathrm{~mm}$ (range $39-40 \mathrm{~mm}$ ) in 6 $(0.4 \%)$ female athletes compared with none of the respective controls. These athletes participated in mixed sports and dynamic sports and were of a similar age compared with athletes with an aortic root below the 99th percentile but had a larger BSA (age: $22.7 \pm 3.7$ vs $19.9 \pm 5.9$ years; $\mathrm{p}=0.116$; BSA: $2.0 \pm 0.15$ vs $\left.1.8 \pm 0.3 \mathrm{~m}^{2} ; \mathrm{p}=0.03\right)$.

Based on $\mathrm{Z}$ scores derived for the general population, 66 athletes $(1.7 \%)$ exhibited a $Z$ score $>2$ of which $21(0.55 \%)$ athletes had a $\mathrm{Z}$ score $>3$. The aortic root diameters in these 66 athletes ranged from 38 to $43 \mathrm{~mm}$.

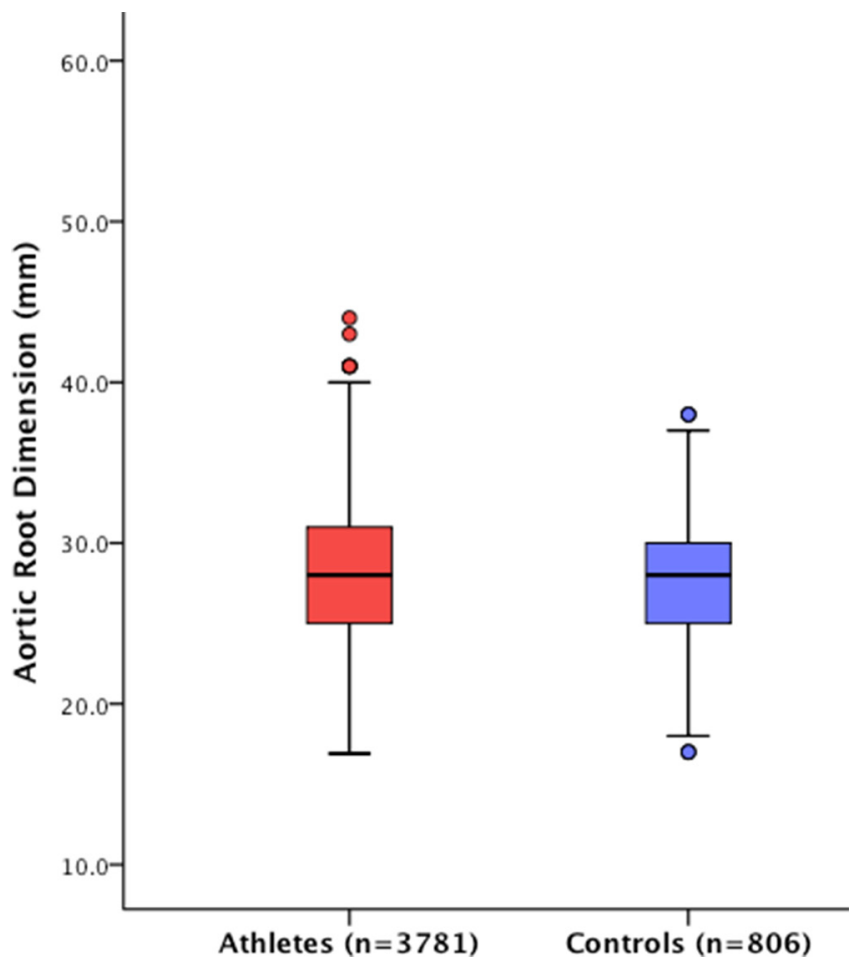

Figure 1 Absolute aortic root dimensions in athletes and controls.
Table 3 Mean aortic root diameter at the level of the sinuses of Valsalva in male and female athletes according to static, endurance and mixed sport

\begin{tabular}{|c|c|c|c|}
\hline Sports category & $\begin{array}{l}\text { Male athletes } \\
(n=2393)\end{array}$ & $\begin{array}{l}\text { Female athletes } \\
(n=1388)\end{array}$ & P valve \\
\hline Static sport & $\begin{array}{l}(n=200) \\
27.7 \pm 3.5 \\
A 0>40 \mathrm{~mm}=1\end{array}$ & $\begin{array}{l}(n=459) \\
26.7 \pm 3.8 \\
A 0>38 \mathrm{~mm}=3\end{array}$ & 0.002 \\
\hline Endurance sport & $\begin{array}{l}(n=584) \\
29.7 \pm 4.0 \\
A 0>40 \mathrm{~mm}=8\end{array}$ & $\begin{array}{l}(\mathrm{n}=337) \\
26.7 \pm 3.7 \\
\text { Ao }>38 \mathrm{~mm}=3\end{array}$ & $<0.001$ \\
\hline Mixed sport & $\begin{array}{l}(n=1609) \\
28.7 \pm 3.9 \\
\text { Ao }>40 \mathrm{~mm}=4\end{array}$ & $\begin{array}{l}(n=592) \\
26.8 \pm 4.0 \\
\text { Ao }>38 \mathrm{~mm}=6\end{array}$ & $<0.001$ \\
\hline$P$ value & 0.462 & 0.600 & \\
\hline
\end{tabular}

Ao, aortic sinus of Valsalva diameter.

Aortic diameter in athletes of African/Afro-Caribbean origin (black athletes)

We performed a separate analysis to assess the impact of ethnicity on aortic root dimensions. Two hundred thirty-four black athletes were compared with 3357 white athletes of a similar age $(19.9 \pm 5.9$ vs $19.9 \pm 5.8$ years; $p=1.000)$. Black athletes had a larger BSA compared with white athletes $(1.91 \pm 0.23$ vs $\left.1.82 \pm 0.25 \mathrm{~m}^{2} ; \mathrm{p}<0.001\right)$. Most black athletes were male $(\mathrm{n}=164,70 \%)$ and engaged in mixed sport (89\%), predominantly soccer $(n=160)$, athletics $(n=35)$, netball $(n=20)$ or basketball $(n=19)$. Black athletes exhibited a slightly larger absolute aortic root diameter compared with white athletes $(29.3 \pm 4.1 \mathrm{~mm}$, range $19-43$ vs $28.3 \pm 4.1 \mathrm{~mm}$, range $17-40 \mathrm{~mm}$; $<<0.001)$, but the 99th percentile values for black male and female athletes were the same as white athletes (40 and $38 \mathrm{~mm}$, respectively). As with white athletes, males revealed a larger aortic root diameter compared with females $(29.3 \pm 3.6 \mathrm{~mm}$; range $(20-43 \mathrm{~mm})$ vs $28.1 \pm 3.7 \mathrm{~mm}$; range $(19-39) \mathrm{mm}, \mathrm{p}<0.001)$.

\section{Determinants of aortic dimensions in athletes}

Multiple-adjusted linear regression analysis including age, BSA, ethnicity, gender, systolic BP, diastolic BP, left atrial diameter, LVEDD LV mass, maximal-LV wall thickness and years of training showed an independent association between aortic root size and BSA and LVEDD (beta standardised coefficients: $\beta=0.255, p<0.001$ and $\beta=0.328, p<0.001$, respectively).

\section{Follow-up assessment in athletes}

Over a mean follow-up of $5 \pm 1.5$ years, none of the male $(n=5)$ or female $(n=6)$ athletes with an enlarged aortic root showed a significant increase in the aortic root diameter (table 4; figure 2). This should be cited as table 3 now. The mean aortic root diameter in male athletes was $40.6 \pm 0.9 \mathrm{~mm}$ at baseline, compared with $40.5 \pm 0.7 \mathrm{~mm}$ at follow-up $(\mathrm{p}=0.111)$. In female athletes, the mean aortic diameter was $38.3 \pm 0.6 \mathrm{~mm}$ at baseline, compared with $38.0 \pm 0.7 \mathrm{~mm}$ at follow-up $(\mathrm{p}=0.275)$. There were no aortic events (aortic dissection, rupture or surgery for aortic root replacement) among the entire athletic cohort, including those with an enlarged aortic diameter.

\section{Intraobserver variability/interobserver variability}

Three hundred twenty random cardiac measurements were repeated on a separate occasion by the first author (SG) and independently by another author (NP). The intraclass correlation coefficient for the intraobserver variability was 0.966 (95\% 
Table 4 Demographics and progression of aortic root diameter in the medium term in athletes with enlarged aortic root diameter (males $>40 \mathrm{~mm}$; females $>38 \mathrm{~mm}$ ) This need to become table 3

\begin{tabular}{lllllll}
\hline Athlete & $\begin{array}{l}\text { Age at } \\
\text { baseline } \\
\text { (years) }\end{array}$ & $\begin{array}{l}\text { BSA } \\
\left(\mathbf{m}^{2}\right)\end{array}$ & Sport & $\begin{array}{l}\text { Baseline } \\
\text { aortic } \\
\text { root } \\
(\mathbf{m m})\end{array}$ & $\begin{array}{l}\text { Aortic root } \\
\text { at final } \\
\text { follow-up } \\
\text { assessment } \\
(\mathbf{m m})\end{array}$ & $\begin{array}{l}\text { Time period } \\
\text { between } \\
\text { first and last } \\
\text { assessment } \\
\text { (years) }\end{array}$ \\
\hline Male & 22 & 2.20 & Rowing & 41 & 41 & 5.7 \\
\hline Male & 26 & 2.14 & Rowing & 41 & 41 & 3.8 \\
\hline Male & 27 & 2.30 & Basketball & 41 & 41 & 3.8 \\
\hline Male & 19 & 2.01 & Cycling & 42 & 42 & 7.8 \\
\hline Male & 20 & 2.21 & Cycling & 42 & 43 & 8 \\
\hline Female & 23 & 1.89 & Football & 39 & 39 & 4.5 \\
\hline Female & 24 & 1.91 & Football & 39 & 39 & 4.8 \\
\hline Female & 18 & 1.97 & Rowing & 40 & 40 & 4.7 \\
\hline Female & 22 & 1.98 & Rowing & 39 & 38 & 4 \\
\hline Female & 24 & 1.99 & Netball & 39 & 39 & 4 \\
\hline Female & 30 & 1.78 & Cycling & 39 & 39 & 4 \\
\hline
\end{tabular}

CI 0.953 to 0.975 ) and the intraclass correlation coefficient for interobserver variability was 0.919 (95\% CI 0.893 to 0.938 ).

\section{DISCUSSION}

This study revealed that athletes show a larger aortic root diameter at the level of sinuses of Valsalva compared with controls. Male athletes revealed a larger aortic root diameter compared with female athletes in all sporting categories and the aortic diameter was marginally greater in black athletes compared with white athletes. The 99th percentile value for the mean aortic diameter in athletic males and females was 40 and $38 \mathrm{~mm}$, respectively, irrespective of ethnicity. We chose the 99th percentile values from this large cohort of athletes as our definition of an enlarged aortic root to minimise the risk of false positive results. The difference in mean aortic root diameter between athletes and controls was only $2 \%$. This difference in magnitude is considerably less than that reported for LV wall thickness and cavity size (10\%-20\%), hence an enlarged aorta should not necessarily be attributed to intensive physical training in all athletes.

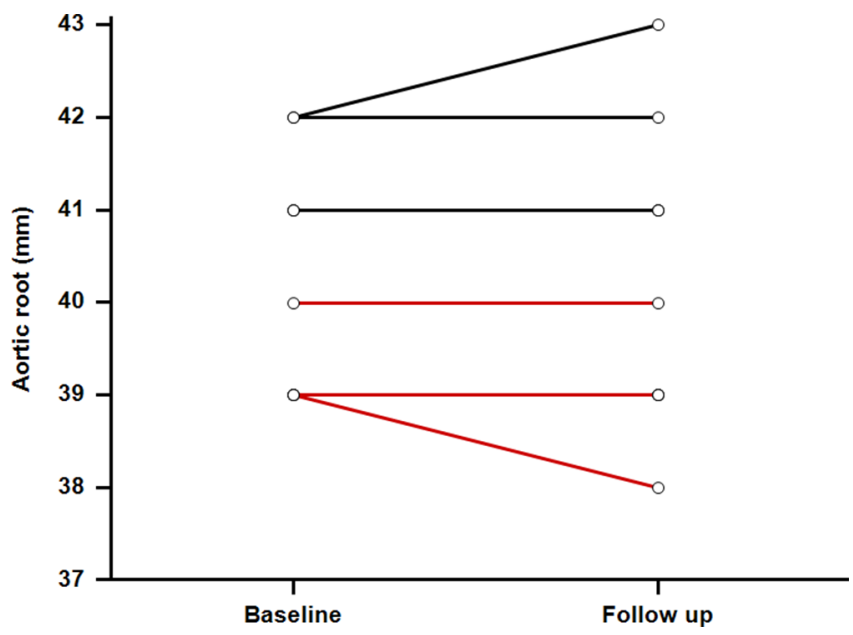

Figure 2 Changes in aortic dimensions over $5 \pm 1.5$ years in athletes with an enlarged aortic root diameter (males $>40 \mathrm{~mm}$; females $38 \mathrm{~mm}$ ) (black lines represent males and red lines represent females).

\section{Determinants of aortic root diameter}

The main determinants of aortic root enlargement in athletes were BSA and LVEDD suggesting that large athletes who trained intensively were more likely to have an enlarged aortic diameter compared with smaller athletes who trained less intensively. However, black ethnicity had no significant influence an enlarged aortic root diameter ( $>40 \mathrm{~mm}$ in males and $>38 \mathrm{~mm}$ in females). Our findings concur with those of Engel et al, who studied aortic diameters in large professional basketball players, 77\% of whom were African-American and showed that the aortic root diameter rarely exceeded $40 \mathrm{~mm} .^{12}$

The impact of sport on aortic dimensions has been a source of great interest and was recently addressed by Boraita et al, ${ }^{4}$ who revealed that absolute aortic dimensions were larger in athletes engaging in sporting disciplines with a high dynamic component. In a meta-analysis of 5580 athletes, ${ }^{3}$ Iskander and Thompson also showed that male endurance athletes had a greater aortic diameter in comparison with strength-trained athletes. Our study also showed that males and females competing in predominantly endurance sports showed a trend towards a larger aortic diameter compared with athletes performing mixed sports and purely static sports.

\section{Mechanism of aortic root enlargement}

The precise mechanism for the aortic root enlargement is unknown. Based on our study and previous large studies, a combination of size, sporting discipline, duration and intensity of training may be contributing factor but BP responses to exercise and genetic factors are also likely important. ${ }^{14}$ Unlike LV wall thickness and the ventricular cavities, the aorta is composed of elastic fibres, which would be expected to recoil in young athletes. Although our ultrasound did not focus precisely on the aortic wall, we suspect that an increase in the thickness of the tunica media may be an important component of aortic size in athletes. A recent rat model investigating the effects of endurance exercise on the heart revealed a thicker tunica media with histological evidence of fibrosis and discontinuity of the elastic layer. $^{13}$

Our study of 3781 athletes is comparable to two previous large studies from Italy ${ }^{1}$ and Spain, ${ }^{4}$ which included 2371 and 3281 athletes, respectively. However, the mean aortic diameter in our cohort was slightly smaller than in both of these studies, most probably because our athletes were younger and had trained for a shorter period. Methodical differences may have also contributed; the Italian study ${ }^{1}$ made measurements derived from M-mode whereas we used two-dimensional measurements. As with the previous studies, very few of our athletes showed an enlarged aortic diameter; however, our 99th percentile for females was $38 \mathrm{~mm}$ compared with $34 \mathrm{~mm}$ in both previous studies. We focused on the aortic root because this is the point of the greatest increase in aortic diameter and also the area that is most vulnerable to dissection. ${ }^{4}$

\section{Absolute aortic root dimensions versus $Z$ scores}

Based on the commonly recommended $\mathrm{Z}$ score, $1.7 \%$ of athletes were deemed to have at least mild aortic root enlargement $(Z$ score $>2$ ) compared with $0.55 \%$ with $\mathrm{Z}$ score $>3$ who would be deemed to have moderate aortic root enlargement. The latter was more comparable with the prevalence of an enlarged aortic diameter based on our 99th percentile value derived from our absolute aortic diameter measurements $(0.3 \%)$, but was still associated with the potential of generating a slightly higher false positive rate. Athletes with an absolute aortic root size 
$>99$ th percentile who also exhibited a $\mathrm{Z}$ score $>3$ did not show progressive aortic root enlargement over the follow-up period.

\section{Longitudinal follow-up}

None of the male or female athletes with an aortic root $>40$ or $>38 \mathrm{~mm}$, respectively, showed significant changes in aortic diameter over a $5 \pm 1.5$ year follow-up period. Haemodynamic load associated with moderate-intensity to high-intensity endurance training did not appear to cause progressive aortic enlargement in our cohort as previously reported. ${ }^{14}$

The AHA/ACC guidelines ${ }^{15}$ recommend that athletic males with an aortic root of $40 \mathrm{~mm}(41 \mathrm{~mm}$ in tall males) and athletic females with an aortic root of $36 \mathrm{~mm}(36-38 \mathrm{~mm}$ in tall females) should only participate in low-intensity competitive sport. Our longitudinal data suggest that male athletes with an aortic diameter up to $43 \mathrm{~mm}$ and female athletes with an aortic diameter up to $41 \mathrm{~mm}$ do not show progressive aortic enlargement over 5 years despite participation in sporting disciplines of a more dynamic nature. Therefore, there is scope for being more liberal in athletes with a slightly enlarged aortic diameter in the future, although annual assessments are recommended.

\section{CONCLUSION}

A small minority of athletes revealed an enlarged aortic diameter. These athletes do not show progressive aortic root enlargement

\section{Key messages}

What is already known on this subject?

- Previous studies report that some young athletes may reveal an enlarged aortic root diameter compared with the general population.

- The significance of aortic root enlargement in young athletes is unknown.

\section{What might this study add?}

- This study investigated 3781 young athletes and showed that the 99th percentile value for the aortic root diameter in males and female athletes was 40 and $38 \mathrm{~mm}$, respectively.

- Approximately $0.3 \%$ athletes had an aortic root diameter exceeding these values (enlarged aortic root diameter); males 41-43 and females $39-40 \mathrm{~mm}$.

- None of the athletes with an enlarged aortic root diameter fulfilled Ghent criteria for Marfan syndrome.

- Athletes with an enlarged aortic root diameter were followed up annually with serial annual echocardiograms over a period of $5 \pm 1.5$ years and failed to show progressive aortic root enlargement during the surveillance process despite ongoing participation in competitive sport.

\section{How might this impact on clinical practice?}

- This study provides upper limits for an aortic root diameter, which are derived from a large cohort of young athletes.

- In the absence of aortopathy, athletes with an enlarged aorta (up to $43 \mathrm{~mm}$ in males and $40 \mathrm{~mm}$ in females) should remain under surveillance and have annual echocardiography while participating in intensive exercise or competitive sport.

- Progressive aortic enlargement $>2 \mathrm{~mm}$ over the ensuing 5 years may be consistent with aortopathy.

- Long-term studies are required to understand the precise significance of an enlarged aortic root in an athlete. over 5 years. Longer prospective studies are required to assess the precise significance of an enlarged aortic root in athletes.

\section{Limitations}

Although athletes with an aortic root diameter $>40 \mathrm{~mm}$ (men) and $>38 \mathrm{~mm}$ (women) were followed up with echocardiography over $5 \pm 1.5$ years, they did not undergo genetic testing to exclude Marfan syndrome. However, a thorough clinical assessment did not give cause to suspect a genetic aetiology. Evaluation of first-degree relatives of athletes with enlarged aortic root diameters may have provided invaluable information in establishing whether aortic root enlargement in our athletes had a familial component. The follow-up of $5 \pm 1.5$ years is considered medium term and may not be entirely sufficient to provide reassurance for the long-term consequences of an enlarged aortic root.

Acknowledgements The authors would like to thank the charitable organisation Cardiac Risk in the Young (CRY) for providing the portable echocardiography equipment and ECG machines used for the study in the UK. The authors would also like to thank Azra Loncarevic-Srmic and Rebecca Howes (UK), who assisted in the collection and collation of data.

Contributors SG, MP and SS: study design, data collection and interpretation, quality control, statistical analysis, manuscript preparation and manuscript revision. NP: data interpretation, quality control, statistical analysis, manuscript preparation and manuscript revision. AM, CS, HD, AHC and RS: data collection and interpretation, and quality control. SS is guarantor.

Funding SG, AM and HD were funded by research grants from CRY and Dr Anne Child was supported by St. George's Hospital NHS Foundation Trust, St. George's University of London, Marfan Trust and Bluff Field Charitable Trust.

Competing interests None declared.

\section{Patient consent for publication Not required.}

Ethics approval Ethical approval was granted by the local research ethics committee in accordance with the Declaration of Helsinki and patients provided oral consent for their anonymised data to be used for this study.

Provenance and peer review Not commissioned; externally peer reviewed.

Data sharing statement All additional unpublished information is kept on a secure server in the institution and only available to the first, second and senior authors of the manuscript.

\section{REFERENCES}

1 Pelliccia A, Di Paolo FM, De Blasiis E, et al. Prevalence and clinical significance of aortic root dilation in highly trained competitive athletes. Circulation 2010;122:698-706.

2 Babaee Bigi MA, Aslani A. Aortic root size and prevalence of aortic regurgitation in elite strength trained athletes. Am J Cardio/ 2007;100:528-30.

3 Iskandar A, Thompson PD. A meta-analysis of aortic root size in elite athletes. Circulation 2013:127:791-8.

4 Boraita A, Heras ME, Morales F, et al. Reference values of aortic root in male and female white elite athletes according to sport. Circ Cardiovasc Imaging 2016;9.

5 Erbel R, Aboyans V, Boileau C, et al. 2014 ESC Guidelines on the diagnosis and treatment of aortic diseases: document covering acute and chronic aortic diseases of the thoracic and abdominal aorta of the adult. The Task Force for the diagnosis and treatment of aortic diseases of the European Society of Cardiology (ESC). Eur Heart J 2014:35:2873-926.

6 Kinoshita N, Mimura J, Obayashi C, et al. Aortic root dilatation among young competitive athletes: echocardiographic screening of 1929 athletes between 15 and 34 years of age. Am Heart J 2000;139:723-8.

7 Cardiac risk in the young. We use cookies to ensure that we give you the best experience on our website. http://www.c-r-y.org.uk

8 Lang RM, Badano LP, Mor-Avi V, et al. Recommendations for cardiac chamber quantification by echocardiography in adults: an update from the American society of echocardiography and the European association of cardiovascular imaging. J Am SoC Echocardiogr 2015;28:1-39

9 Lang RM, Bierig M, Devereux RB, et al. Recommendations for chamber quantification. Eur J Echocardiogr 2006;7:79-108.

10 Devereux RB, de Simone G, Arnett DK, et al. Normal limits in relation to age, body size and gender of two-dimensional echocardiographic aortic root dimensions in persons $\geq 15$ years of age. Am J Cardiol 2012;110:1189-94. 
11 De Paepe A, Devereux RB, Dietz HC, et al. Revised diagnostic criteria for the marfan syndrome. Am J Med Genet 1996;62:417-26.

12 Engel DJ, Schwartz A, Homma S. Athletic cardiac remodeling in US professional basketball players. JAMA Cardiol 2016;1:80-7.

13 Rubies C, Batlle M, Castillo N, et al. Effects of long-term very high intensity exercise on vascular remodelling in an animal model. 2016.

14 D'Andrea A, Cocchia R, Riegler L, et al. Aortic root dimensions in elite athletes. Am J Cardiol 2010;105:1629-34.
15 Hiratzka LF, Bakris GL, Beckman JA, et al. 2010 ACCF/AHA/AATS/ACR/ASA/SCA/ SCAI/SIR/STS/SVM guidelines for the diagnosis and management of patients with thoracic aortic disease: a report of the American college of cardiology foundation/ american heart association task force on practice guidelines, american association for thoracic surgery, american college of radiology, american stroke association, society of cardiovascular anesthesiologists, society for cardiovascular angiography and interventions, society of interventional radiology, society of thoracic surgeons, and society for vascular medicine. Circulation 2010;121:e266-369. 